\title{
Análisis prospectivo y escenario de pymes panaderas, caso megasuperior en la provincia Tsachilas - Ecuador ${ }^{*}$
}

\author{
Recibido: 10 de agosto de 2020 - Aprobado: 16 de octubre de 2020 \\ https://doi.org/10.22395/seec.v23n55a9
}

\author{
Félix Pilay Toala** \\ Lady Gallardo Montes ${ }^{* * *}$ \\ Lissette Vásquez Muñoz ${ }^{* * *}$
}

\section{RESUMEN}

El objetivo de este estudio es dar a conocer los beneficios del uso de la disciplina prospectiva estratégica para el correcto direccionamiento y toma de decisiones en las empresas panaderas, caso megasuperior. La metodología aplicada cuali-cuantitativa con investigación descriptiva, utilizó un software Mic Mac para analizar la relación entre las variables Mactor que establece la identificación de actores para convergencias y divergencias; se aplicaron a expertos 100 encuestas Delphi de una muestra por conveniencia para obtener probabilidades simples y condicionales. La obtención de factores claves se efectuó a través del diagnóstico para la información de fuentes primarias y secundarias, se procedió a identificar escenarios en donde se cumplieron los objetivos, se verificó procedimientos de ejecución en el árbol de pertinencias. Se contrastó con la teoría de Godet para el procesamiento de datos que permitieron reducir incertidumbres ante las probabilidades subjetivas. Los resultados evidenciaron cambios ocurridos y originados por el alto endeudamiento, deficiente política de ventas y restricción de mercado. Los hallazgos mediante el método Mic Mac, existencia de nodos críticos por el alto nivel de dependencia, baja rotación de inventario y escasa fuerza de ventas. Conclusiones, que el endeudamiento supera los límites financieros saludables.

\section{PALABRAS CLAVE}

Escenarios; Toma de Decisiones; Nodos Críticos; Panaderas; Inventario.

\section{CLASIFICACIÓN JEL} L88, M1 y M21

\section{CONTENIDO}

Introducción; 1. Metodología; 2. Resultados; 3. Conclusiones; Bibliografía.

\footnotetext{
Artículo de investigación. Proyecto de grado en administración de empresas, ejecución 2019, Línea de investigación Administración eficiente y eficaz de las organizaciones para la competitividad sostenible local y global, tema "Análisis prospectivo y escenarios probabilísticos de la empresa "megasuperior", localizada en la ciudad de Santo Domingo. Tutor. Mg. Félix Pilay Toala"

.- Doctor. Profesor a tiempo completo de la Pontificia Universidad Católica del Ecuador, Santo Domingo. Correo electrónico: ptfs@pucesd.edu.ec. Orcid: https://orcid.org/0000-0001-7376-3197

... Ingeniera Comercial, Pontificia Universidad católica del Ecuador, Santo Domingo. Correo electrónico: Itgallardom@pucesd.edu.ec. Orcid: https://orcid.org/0000-0001-8217-9865

.... Ingeniera Comercial, Pontificia Universidad católica del Ecuador, Santo Domingo. Correo electrónico: ljvasquezm@pucesd.edu.ec. Orcid: https://orcid.org/0000-0001-7406-3985
} 


\title{
PROSPECTIVE ANALYSIS AND SCENARIO OF BAKERY SMEs, MEGASUPERIOR CASE, IN THE PROVINCE TSACHILAS - ECUADOR ABSTRACT
}

\begin{abstract}
The research was a degree thesis, the objective was to publicize the benefits of the use of prospective discipline for the correct direction and decision making in bakery companies, megasuperior case. The applied methodology is quali-quantitative with descriptive research, it used a MIC MAC software to analyze the relationship between the MACTOR variables that establishes the identification of actors for convergences and divergences, 100 Delphi surveys of a convenience sample were applied to experts to obtain probabilities simple and conditional. The obtaining of key factors was carried out through the diagnosis for the information from primary and secondary sources, we proceeded to identify scenarios where the objectives were met, execution procedures were verified in the pertinence tree. It was contrasted with Godet's theory for data processing that allowed reducing uncertainties in the face of subjective probabilities. The results showed changes that occurred and originated from high indebtedness, poor sales policy and market restriction. The findings using the MIC MAC method, existence of critical nodes due to the high level of dependency, low inventory turnover and low sales force. Conclusions, that indebtedness exceeds healthy financial limits.
\end{abstract}

\section{KEYWORDS}

Scenarios; Decision Making; Critical Nodes; Bakers; Inventory.

\section{CLASSIFICATION JEL}

L88, M1 and M21

\section{CONTENT}

Introduction; 1. Methodology; 2. Results; 3. Conclusions; Bibliography.

\section{ANÁLISE PROSPECTIVA E CENÁRIO DE PADARIAS, CASO MEGASUPERIOR, NA PROVÍNCIA DE TSACHILAS - EQUADOR \\ RESUMO}

A pesquisa foi uma tese de graduação, o objetivo foi divulgar os benefícios da utilização da disciplina prospectiva para o correto direcionamento e tomada de decisão em empresas de panificação, caso megassuperior. A metodologia aplicada é quali-quantitativa com pesquisa descritiva, utilizou um software MIC MAC para analisar a relação entre as variáveis MACTOR que estabelece a identificação de atores para convergências e divergências, 100 inquéritos Delphi de uma amostra de conveniência foram aplicados a especialistas para obter probabilidades simples e condicional. A obtenção dos factores-chave foi efectuada através do diagnóstico das informações das fontes primárias e secundárias, procedeu-se à identificação de cenários onde os objectivos foram cumpridos, foram verificados os procedimentos de execução na árvore de pertinência. Foi contrastado com a teoria de Godet para processamento de dados que permitia reduzir as incertezas diante das probabilidades subjetivas. Os resultados evidenciaram mudanças ocorridas e decorrentes do alto endividamento, má política de vendas e restrição de mercado. Os achados utilizando o método MIC MAC, existência de nós críticos devido ao alto nível de dependência, baixo giro de estoque e baixa força de vendas. Conclusões, esse endividamento ultrapassa limites financeiros saudáveis.

\section{PALAVRAS-CHAVE}

Cenários; Tomando Uma Decisão; Nós Críticos; Padeiros; Inventário.

\section{CLASSIFICAÇÃO DE JEL:}

L88, M1e M21

\section{CONTEÚDO}

Introdução; 1. Metodologia; 2. Resultados; 3. Conclusões; Bibliografia. 


\section{INTRODUCCIÓN}

El trabajo investigativo tiene como propósito la elaboración de un análisis prospectivo y escenarios probabilísticos de la empresa Megasuperior, dedicada a la industria de la panificación, ubicada en Santo Domingo, Ecuador, utilizando la metodología del Mic Mac y del Mactor para determinar las divergencias y convergencias entre actores y los objetivos estratégicos, y con ello determinar los posibles escenarios futuros mediante la aplicación del método Smic Prob Expert. La metodología empleada es el enfoque cuali-cuantitativo basada en el modelo planteado por Mishel Godet.

Se busca, mediante la investigación, dar a conocer los beneficios que trae consigo el uso de la disciplina de prospectiva estratégica para el correcto direccionamiento de las pequeñas y medianas empresas del país, debido a que por lo general este tipo de organizaciones suelen ser dirigidas de manera empírica, lo que dificulta el desarrollo en un entorno competitivo; actualmente existen diversas herramientas que se pueden utilizar para desplegar una visión del futuro que permita tomar medidas para entender y aportar a la resolución de posibles problemas futuros y alcanzar los objetivos planteados.

En América Latina y el Caribe las micro, pequeñas y medianas empresas [Mipyme] son las que presentan mayor capacidad para generar empleo al dedicarse desde actividades de subsistencia, comercialización, hasta la exportación; sin embargo, el desarrollo administrativo se efectúa bajo un sistema informal desde sus primeros años, siendo complicado conservarse en el mercado a largo plazo y presentando un bajo nivel de productividad (Organización Internacional de Trabajo, 2016).

En el 2017 se desarrolló una investigación a organizaciones Latinoamericanas, explicando que la prospectiva estratégica cuenta con herramientas informáticas que permiten a la organización visualizar y construir alternativas futuras, obteniendo como resultado un total de 17 variables estratégicas, 9 actores principales y 64 posibles escenarios, donde se seleccionó el escenario alternativo, que permite una mejor toma de decisiones para el cumplimiento de los objetivos estratégicos (Armijos et al., 2017).

En Ecuador se realizó un estudio sobre el "procedimiento para incrementar la gestión competitiva de las pequeñas y medianas empresas comerciales ecuatorianas" (Quiñonez, 2017), las empresas comerciales registradas en el país, representan el $40 \%$ de concentración y contribuyen el $65 \%$ del empleo, por tanto el estudio de la empresa Megasuperior se vuelve relevante para determinar su gestión competitiva, y buscar resultados que permitan que las empresas locales mejoren la competitividad, la satisfacción de los clientes, emprendan una adecuada toma de decisiones y oportunidades. 
Megasuperior se encuentra en la Provincia de Santo Domingo de los Tsáchilas, es una empresa considerada como pequeña empresa o Pyme, ubicada dentro del sector panadero, cuenta con varias líneas de comercialización, como la de materia prima, implementos e insumos; su actividad principal comprende la compra y venta al por mayor y menor de materia prima como es la "harina superior" y, además, varios insumos como las cepa de levadura y otros, al mismo tiempo, comercializa varios tipos de maquinarias que son usadas en la industria de la panificación. "La línea de productos se refiere a un grupo de productos que tienen una relación directa entre sí, puede ser por sus características físicas, utilidad o segmento. Dentro del mundo de la mercadotecnia, es una estrategia que ofrece diferentes beneficios" (Mesa Editorial Merca2.0, 2015).

Para realizar un análisis prospectivo, en el caso de la empresa Megasuperior, se consideró necesario emplear las herramientas desarrolladas por Michael Godet, estás facilitan la realización del análisis. Así como también contar con la participación de expertos del sector para captar información y direccionar de manera correcta el análisis, a más de esto, lo imprescindible es contar con información confiable brindada por personas que laboran cada día en este sector.

De manera previa se realiza un listado de las variables internas y externas que tienen gran impacto para la empresa de modo minucioso, es necesario considerar la opinión de expertos como información confiable, por ser conocedores de la gestión empresarial de empresas similares a Megasuperior; después, se procede a la redacción para determinar lo que ocurre en la actualidad e identificar el indicador medible. La formulación de las variables es la orientación para realizar el plan de acción, que se determina en el árbol de pertinencias con los objetivos a largo plazo (Campos y Salas, 2016).

El centro de las fuerzas competitivas, que permite analizar el grado de competitividad entre las empresas pequeñas y medianas dedicadas a la actividad panadera - caso de Megasuperior - , es la comercialización de producto, como la harina, y subproductos, como molturación de trigo, harina de otros cereales, entre otros, que conlleva a las pequeñas panificadoras a implementar estrategias positivas o negativas según el entorno; una de las variables estratégicas externas identificada, es la competencia desleal en la comercialización de los productos y subproductos. "Los cambios de estrategia que ponga en práctica una empresa podrían dar lugar a represalias como reducción de precios, mejoras a la calidad, introducción de nuevas características en los productos, ofrecimiento de servicios, extensión de garantías y aumento de publicidad" (David, 2013, p. 76). 
La competencia desleal entre las empresas panaderas con mayor énfasis se genera en las cepas de levadura, que se comercializan y que se las utiliza como elemento fundamental para elaborar pan o productos de panadería. Las levaduras son comercializadas en cinco tipos: seca, instantánea, líquida, desactivada y especial; cumpliendo dos funciones centrales, tales como producir gas para oxigenar el pan o la masa, y también contribuir a la etapa de maduración, atravesando por la fermentación y con ello obtener como resultado una masa esponjada (Lezcano, 2010; Payehuanca y Matos, 2011).

Todas las empresas se ven en la obligatoriedad de competir entre sí, en la carrera de generar valor que les permita subsistir en el mercado, para ello deben cumplir con su principal necesidad: satisfacer a sus clientes de manera eficiente. "Las fuerzas competitivas más fuertes determinan la rentabilidad de un sector y se transforman en los elementos más importantes de la elaboración de la estrategia. La fuerza más relevante, sin embargo, no siempre es obvia" (Porter, 2008).

Realizado el diagnóstico estratégico de la empresa Megasuperior, así como el procesamiento de la información obtenida mediante sistemas informáticos del Lipsor (Laboratory for Investigation in Prospective Strategy and Organisation), se analizó el nivel de influencia y dependencia de las variables estratégicas y actores, identificando la relación competitiva con el cumplimiento de objetivos. "Su objetivo es proponer orientaciones estratégicas y acciones basadas en las competencias de la organización en función de los escenarios de evolución de su entorno" (Godet y Durance, 2011, p. 35).

\section{METODOLOGÍA}

Contiene la descripción y argumentación de las importantes decisiones metodológicas adoptadas según los objetivos, las preguntas y las variables de la investigación propuesta y las posibilidades del indagador. La claridad en el tipo de enfoque y estructura metodológica fue condición necesaria para asegurar la validez de la investigación.

El estudio se desarrolló a partir de dos enfoques: el cuantitativo con la recolección de datos a través de encuestas aplicadas a expertos, propuesta que se basa en la investigación descriptiva al realizar el diagnóstico situacional de Megasuperior. "Busca especificar propiedades, características y rasgos importantes de cualquier fenómeno que se analice. Describe tendencias de un grupo o población" (Sampieri, Collado, y Baptista, 2010, p. 80). 
Desde la perspectiva del enfoque cuantitativo se procede a la aplicación de un instrumento para obtener datos de expertos, lo que permitió recoger y analizar información numérica, procesada en un software con la finalidad de obtener resultados porcentuales acertados con un nivel de probabilidad.

En el enfoque cuantitativo se aplicó la investigación descriptiva, se recopiló información de manera independiente o conjunta al obtener datos sobre la situación actual interna y externa de la empresa, para determinar las variables y conocer las causas que impiden el desenvolvimiento competitivo empresarial de Megasuperior.

Del enfoque cualitativo se encaminó a la recolección de datos que no tuvieran relación con parámetros numéricos y de esta manera descubrir preguntas de investigación en el proceso de interpretación. Mediante este instrumento se obtuvo la recolección de datos por medio de entrevistas con el personal administrativo, para identificar y realizar el diagnóstico de la situación actual de la empresa.

Para el desarrollo del análisis prospectivo, relacionado con Megasuperior, se consideró los tres software propuestos por Godet y Durance (2011): análisis estructural (Mic Mac), análisis de las estrategias de actores (Mactor) y determinación de escenarios probabilísticos (Smic Prob Expert), de acuerdo al número de variables claves presentadas en el plano de influencias y dependencias.

\subsection{Población y muestra}

Según Sampieri, Collado y Baptista (2010) la población es el conjunto de todos los elementos que poseen una serie de especificaciones, por ello se consideró a expertos en el sector panadero y distribuidores de materia prima, insumos y maquinaria de Panaderías establecidos en la Provincia de Santo Domingo de los Tsáchilas y a los stakeholders de Megasuperior; según datos del Instituto Ecuatoriano de Estadísticas y Censos (2010) existen 7.957 establecimientos a nivel nacional.

Se aplicó un muestreo aleatorio no probabilístico por conveniencia a 100 expertos en el área panadera y distribuidores, representados por gerentes y directores de las empresas con actividades comerciales al por mayor y menor de materia prima, insumos y maquinaria de Panadería, además del personal de Megasuperior.

\subsection{Análisis de datos}

Se analiza a la empresa panadera Megasuperior a través del procesamiento de datos secundarios internos, como estados financieros. En este sentido, por medio de entrevistas se desarrolló la cadena de valor, estructura organizacional, mapa 
de procesos y ciclo de vida del producto; también a través de fuentes secundarias externas como prensa virtual, revistas y organizaciones nacionales e internacionales.

Con respecto a la perspectiva externa se procesó la información obtenida de fuentes secundarias externas, como el Instituto Nacional de Estadísticas y Censos (INEC), Plan Nacional del Buen Vivir, Código Orgánico de la Producción, Comercio e Inversiones, Organización Internacional del Trabajo (OIT) e información en prensa virtual como el Comercio, Revista Líderes y Ekos.

\subsection{Análisis mediante software Mactor y Smic Prob Expert}

En este método de análisis Mactor se determinó los tipos de actores de acuerdo a las posiciones en el plano de influencias y dependencias, así como, la relación de convergencias y divergencias entre los involucrados, al realizar los objetivos estratégicos en el juego de actores, se procedió con la matriz de actores/objetivos $2 \mathrm{Mao}$, histograma de la implicación de los actores sobre los objetivos 2Mao, histograma de la movilización de los actores sobre los objetivos $3 \mathrm{Mao}$ y el histograma de relaciones de fuerza MMIDI.

En el Software Smic Prob Expert, con los datos obtenidos de las encuestas Delphi aplicadas a expertos en el sector comercial de ventas al por mayor y menor, para conocer el criterio sobre el nivel de cumplimiento de los objetivos propuestos en el juego de actores, desde tres aspectos: condicionales simples, positivos y negativos. Como resultado se obtiene la combinación binaria de los seis eventos futuros, representados en el histograma de extremums, interpretando tres escenarios; tendencial, apuesta y alternativo.

\section{RESULTADOS}

Para el análisis situacional interno y externo de la empresa Megasuperior, se utilizó herramientas de gestión administrativa, de marketing y software Mactor y Smic Prob Expert que permitieron identificar las fortalezas y debilidades de la organización. Por lo extenso del análisis, se reseñarán las cuestiones centrales y relevantes de la información obtenida del proceso investigativo.

El análisis externo se desarrolló desde lo macroambiente, que son fuerzas al ser consideradas como factores políticos, económicos, sociales, tecnológicos y ambientales, y el microambiente, que comprende las fuerzas competitivas de Michael Porter. Desde lo interno se consideró los diferentes elementos o variables que existen dentro de la empresa, determinando las principales fortalezas y debilidades en cada departamento desde el nivel directivo hasta el operacional. 
En el análisis financiero realizados en la empresa Megasuperior se puede apreciar que durante tres años consecutivos (2016, 2017 y 2018) la liquidez se presentó favorable desde 1,11 - 1,18 y 2,41; más sin embargo, el nivel de endeudamiento de una unidad empresarial presenta cifras por encima de los parámetros saludables, desde la perspectiva financiera correspondiente al $69 \%$, con un margen de rentabilidad del 1,5\%; con respecto al ciclo del dinero correspondiente al año 2018 se determinó que el resultado es desfavorable, porque debe cancelar a los acreedores cada 11 días y el retorno de efectivo de las ventas es cada 38 días, entonces, existe una diferencia de 27 días. Para el cumplimiento de las actividades comerciales, el financiamiento procede de préstamos bancarios a largo plazo $(\$ 83.837,31)$.

\subsection{Análisis estructural con el método Mic Mac.}

Se procede con el uso de la herramienta tecnológica para determinar las variables estratégicas, empleando el método Mic Mac (Matriz de Impactos Cruzados Multiplicación Aplicada a una Clasificación) para la identificación de los nudos críticos que tienen mayor influencia y dependencia a partir de los cuales se van a estructurar los objetivos a largo plazo.

La relación entre variables fue procesada mediante una matriz de análisis estructural de doble entrada, con lo cual se estableció una calificación cualitativa, con un total de 1260 preguntas, aplicando la fórmula del sistema $\left(n^{*} n-1\right)$. En el plano correspondiente a la Figura 1 se observa la posición de las 36 variables estratégicas obtenidas del diagnóstico situacional, es relevante identificar los nudos críticos ubicados en la zona de enlace por el alto nivel de dependencia e influencia, siendo: Líneas de productos (LDP), Cobertura de distribución de mercado (CDIS), Rotación del inventario (ROTI), Logística de distribución (LOG), Precios competitivos (PRCO) y Administración de la fuerza de ventas (ADFV).

Las variables pueden clasificarse de acuerdo a la posición en el plano desde: determinantes, del entorno, autónomas, reguladoras, de resultado, secundarias y objetivo; con respecto a las de entrada se puede evidenciar cuatro elementos: Alianzas estratégicas (ALE), Inversión en gestión de innovación (IGIN), Créditos para proyectos de inversión en Mipymes (CPIM) y endeudamiento (EDD), la presencia de estos nudos es importante para las gestiones empresariales con otras instituciones y su financiamiento. 
Figura 1. Plano de influencias y dependencias indirectas.

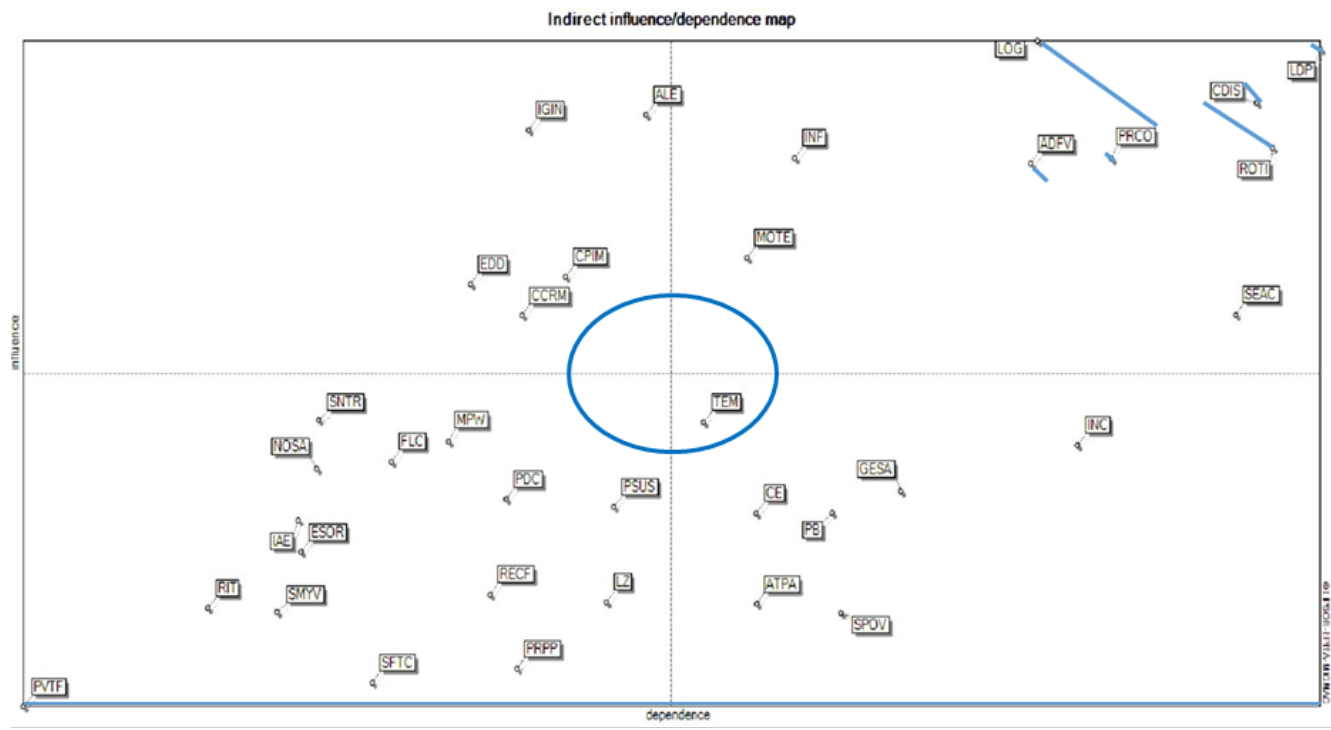

Fuente: Laboratorio de Investigación de Prospectiva Estratégica y Organización. (2016). Mactor (Versión 5.3.0) [Windows]. Francia. Elaboración propia

\subsection{Software método Mactor.}

Mediante el Método Mactor (Matriz de Alianzas y Conflictos: Tácticas, Objetivos y Recomendaciones) se valora las relaciones de fuerza entre los actores, analizando las convergencias y divergencias con respecto a determinados retos u objetivos. El proceso inicia con el listado de los actores, examinando el medio de acción de quienes se encuentran a favor y en contra, para posteriormente armar las jugadas.

\subsection{Matriz de actores por actores.}

Por medio de la matriz de influencias directas se establece la relación existente entre los 31 involucrados, valorando el accionar de cada uno y calculando la correlación de fuerza existente. Los resultados obtenidos permiten realizar un análisis al juego de actores tanto de manera directa como indirectamente en cada reto estructurado con relación a las variables claves.

\subsection{Plano de influencias y dependencias entre actores.}

La clasificación ordenada de los involucrados, ubicados en el plano de influencias y dependencias fueron realizados de acuerdo al nivel de motricidad y dependencia, 
por tanto, se puede evidenciar que ocupan posiciones en cada cuadrante con la siguiente denominación: actores dominantes, actores repetidores, actores autónomos y actores dominados. La influencia se identifica a través de una escala desde $0 \mathrm{a}$ 4 establecidas mediante el software Mactor: (0) El actor (Ai) no tiene medios de acción sobre el actor (Aj), (1) El actor (Ai) influye en los procesos que desempeña su similar comparativo (Aj), (2) El actor (Ai) cuestiona los proyectos del actor (Aj), (3) El actor (Ai) puede cuestionar las misiones del actor (Aj), (4) El actor (Ai) puede cuestionar la existencia del actor (Aj), como se observa en la figura 2.

Los stakeholders o los involucrados que poseen un alto nivel de influencia y dependencia son los repetidores como: (GER) Gerente y (MEGS) Megasuperior, mientras que los dominantes son: (MINT) Ministerio del Trabajo, (IESS) Instituto Ecuatoriano de Seguridad Social, (ARCSA) Agencia Nacional de Regulación, Control y Vigilancia Sanitaria, (INSF) Instituciones Financieras, (JEFV) Jefe de Ventas y (ASESJ) Asesor Jurídico.

Figura 2. Plano de influencias y dependencia entre actores.

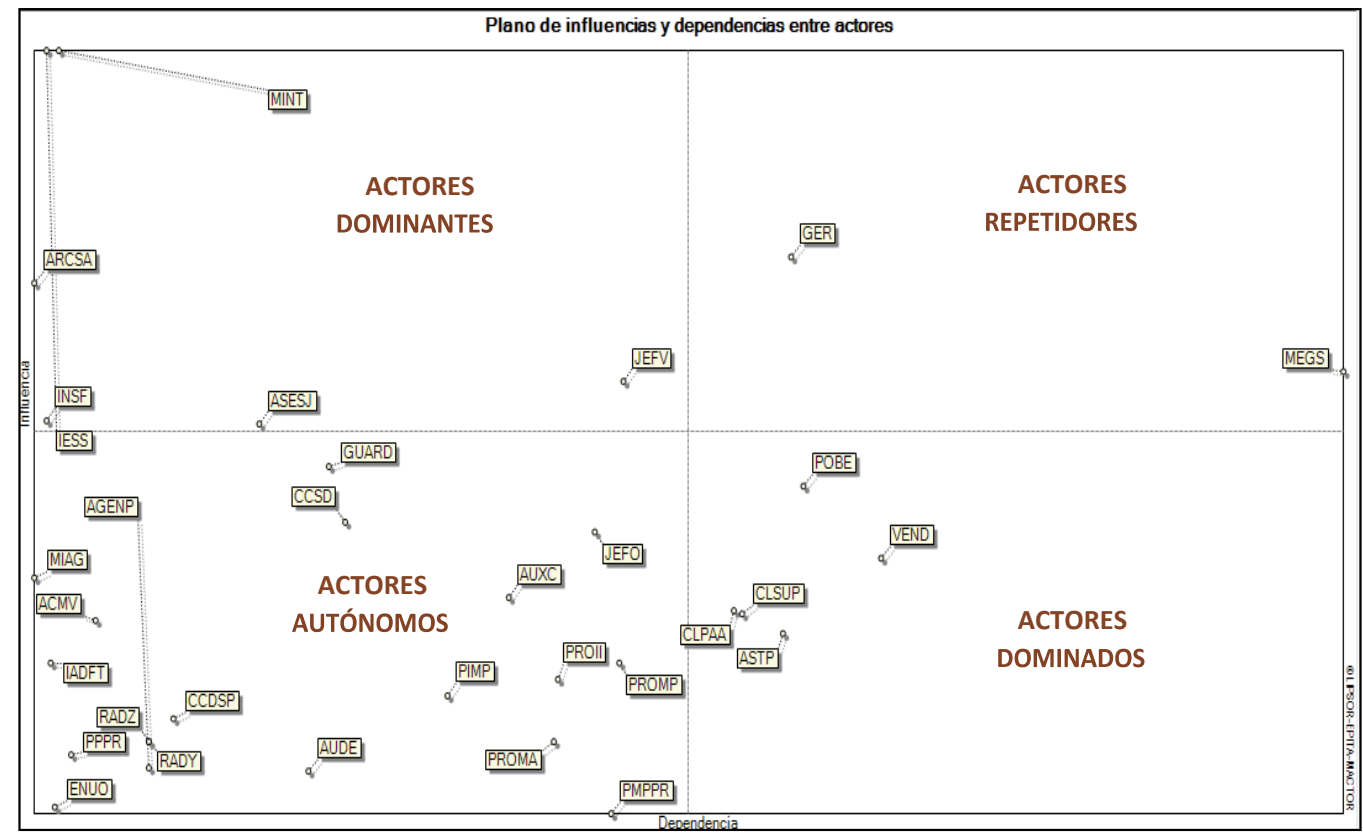

Fuente: Laboratorio de Investigación de Prospectiva Estratégica y Organización. (2016). Mactor (Versión 5.3.0) [Windows]. Francia. Elaboración propia 


\subsection{Convergencia entre autores.}

Mediante la convergencia se identifican el nivel de correlación de interés entre los actores para el cumplimiento de los objetivos estratégicos, propuestos por Megasuperior. Considerando como actores aliados a (GER) Gerencia e (INSF) Instituciones Financieras, así como (GER) Gerencia y (JEFV) Jefe de Ventas, quienes favorecen el cumplimiento de las actividades comerciales.

\subsection{Objetivos.}

Posteriormente se formularon seis objetivos estratégicos relacionados con las variables claves:

1) Gestionar una línea de harinas industriales con trigos importados bajo la certificación World Basc Organization en un lapso de 2 años.

2) Establecer un centro de distribución con líneas de productos para panadería, pastelería y repostería en la ciudad de Esmeraldas en un período de 2 años.

3) Incrementar a 39,47 veces la rotación del inventario en un lapso de 3 años.

4) Implementar un Sistema de Voice Picking en los procesos de almacenaje y distribución de mercadería en un período de 2 años y medio.

5) Instalar una Escuela de Panadería, Repostería y Pastelería en la ciudad de Santo Domingo en un lapso de 3 años.

6) Implementar un plan de capacitación integral continua a la fuerza de ventas, en un lapso de 2 años.

\subsection{Matriz de actores/objetivos 2Mao implicación y movilización 3Mao.}

A partir de la matriz de actores sobre objetivos $2 \mathrm{Mao}$, se identifica el compromiso que tienen los involucrados para el cumplimiento de gestionar una línea de harinas industriales con trigos importados bajo la certificación World Basc Organization en un lapso de 2 años, con 30 posiciones a favor y 5 en contra. La movilización de los actores sobre los objetivos se representa en el histograma 3Mao, en el cual es posible gestionar una línea de harinas industriales con trigos importados bajo la certificación World BASC Organization, 25 posiciones valoradas a favor y 5,8 en contra. 


\subsection{Método de Impacto Cruzados Probabilísticos.}

\subsubsection{Eventos probabilísticos.}

Como se observa en la tabla 1 al identificar las variables claves o nudos críticos se determinaron objetivos a largo plazo que permitieron el desarrollo empresarial de Megasuperior relacionando la situación actual con nudos críticos y objetivos estratégicos, después se procedió a establecer las probabilidades simples y condicionales.

\section{Tabla 1. Hipótesis.}

\begin{tabular}{|c|c|c|c|}
\hline \multicolumn{4}{|c|}{ EVENTOS PROBABILÍSTICOS } \\
\hline $\mathrm{N}^{\circ}$ Evento & Variable & Objetivo $(\mathrm{H})$ & Situación Actual \\
\hline E1 & $\begin{array}{l}\text { Línea de } \\
\text { productos }\end{array}$ & $\begin{array}{l}\text { Gestionar una línea de harinas indus- } \\
\text { triales con trigos importados bajo la } \\
\text { certificación World Basc } \\
\text { Organization en un lapso de } 2 \text { años. }\end{array}$ & $\begin{array}{l}\text { Megasuperior posee una } \\
\text { línea de harinas de la marca } \\
\text { Ecuatoriana Superior. }\end{array}$ \\
\hline E2 & $\begin{array}{l}\text { Cobertura de } \\
\text { distribución } \\
\text { de mercado }\end{array}$ & $\begin{array}{l}\text { Establecer un centro de distribución } \\
\text { con líneas de productos para panadería, } \\
\text { pastelería y repostería en la ciudad de } \\
\text { Esmeraldas en un período de } 2 \text { años. }\end{array}$ & $\begin{array}{l}\text { La empresa no posee } \\
\text { centros de distribución fuera } \\
\text { de Santo Domingo. }\end{array}$ \\
\hline E3 & $\begin{array}{l}\text { Rotación del } \\
\text { inventario }\end{array}$ & $\begin{array}{l}\text { Incrementar a 39,47 veces la rotación del } \\
\text { inventario en un lapso de } 3 \text { años. }\end{array}$ & $\begin{array}{l}\text { La rotación del inventario } \\
\text { es de } 32,79 \text { veces en el año, } \\
\text { equivalente a } 11 \text { días. }\end{array}$ \\
\hline E4 & $\begin{array}{l}\text { Logística de } \\
\text { distribución }\end{array}$ & $\begin{array}{l}\text { Implementar un Sistema de Voice Picking } \\
\text { en los procesos de almacenaje y distri- } \\
\text { bución de mercadería en un período de } 2 \\
\text { años y medio. }\end{array}$ & $\begin{array}{l}\text { Los procesos de almacenaje } \\
\text { y distribución de mercadería } \\
\text { son controlados con hojas } \\
\text { de trabajo. }\end{array}$ \\
\hline E5 & $\begin{array}{l}\text { Precios } \\
\text { competitivos }\end{array}$ & $\begin{array}{l}\text { Instalar una Escuela de Panadería, } \\
\text { Repostería y Pastelería en la ciudad de } \\
\text { Santo Domingo, un lapso de } 3 \text { años. }\end{array}$ & $\begin{array}{l}\text { La entidad solo ofrece } \\
\text { talleres o eventos para el } \\
\text { sector panadero. }\end{array}$ \\
\hline E6 & $\begin{array}{l}\text { Administra- } \\
\text { ción de la } \\
\text { fuerza de } \\
\text { ventas }\end{array}$ & $\begin{array}{l}\text { Implementar un plan de capacitación } \\
\text { integral continua a la fuerza de ventas, en } \\
\text { un lapso de } 2 \text { años. }\end{array}$ & $\begin{array}{l}\text { El departamento de ventas } \\
\text { no posee un plan de capaci- } \\
\text { tación integral continua para } \\
\text { vendedores. }\end{array}$ \\
\hline
\end{tabular}

Fuente: Creación propia. Elaborado por: Autores 


\subsubsection{Probabilidades simples.}

Realizadas las encuestas Delphi a expertos en el área panadera y distribuidores, representados por gerentes y directores de las empresas con actividades comerciales al por mayor y menor de materia prima, insumos y maquinaria de panadería, se procede a ingresar los datos al sistema Smic ProbExpert, obteniendo como resultado las probabilidades simples en relación a los seis eventos propuestos bajo los siguientes resultados: 1-GLHI (Gestionar línea de harinas industriales), (0,44), 2- EDCD (Establecer un centro de distribución), (0,57), 3-INCRI (Incrementar rotación de inventario), (0,509), 4-ISVP (Implementar Sistema de Voice Picking), (0,461), 5- IEPDC (Instalar escuela de panadería, repostería y pastelería), $(0,533)$ y 6- IPCIFV (Plan de capacitación integral a fuerza de ventas), $(0,525)$.

\subsubsection{Descripción de escenarios.}

El escenario tendencial con la probabilidad relativa más alta del 31,9\% establece que la Pymes tendrá dificultades para la implementación de un plan de capacitación integral a la fuerza de ventas, incluso el alto endeudamiento (69 \%) impide la implementación de un Sistema de Voice Picking en los procesos de almacenaje y distribución, perjudicando la planificación logística, esto dificulta establecer un centro de distribución en Esmeraldas, complicando el incremento de la rotación del inventario, así como la gestión de una línea de harinas industriales con trigos importados y ante la limitada diversidad de productos la instalación de una escuela de panadería, repostería y pastelería, es complicado.

El escenario apuesta con un 27,7 \% se ubica en segundo lugar, con una probabilidad relativa en todos los objetivos de Megasuperior, al mismo tiempo se determina que a pesar de que tuvo el $69 \%$ de endeudamiento en el 2018, se incrementó la rotación del inventario, esto favoreció la gestión de una línea de harinas industriales con trigos importados, permitiendo la diversidad de productos al establecer un centro de distribución en Esmeraldas, promoviendo la implementación de un plan de capacitación integral a la fuerza de ventas y la instalación de una escuela de panadería, pastelería y repostería; finalmente, para un control adecuado de los productos se implementó un Sistema de Voice Picking en los procesos de almacenaje y distribución.

\section{CONCLUSIONES}

Con respecto al diagnóstico situacional de Megasuperior, se destaca un endeudamiento del $69 \%$ que sobrepasa los parámetros saludables financieros, esto se debe a la adquisición de obligaciones financieras porque debe cubrir las deudas 
cada 11 días mientras que el retorno de ventas es cada 38 días, siendo desfavorable; con respecto a la liquidez es del 2,41 \% y la rentabilidad del 1,5\%, relativa al último año. Aunque la empresa posee un sólido equipo de ventas y servicio postventa, el planteamiento de objetivos y metas sin fundamentos estratégicos ha generado inconvenientes en la planificación logística. Al momento de realizar las entrevistas la limitante fue la dificultad para aplicarlas a algunos colaboradores.

A partir del análisis estructural con el método Mic Mac de la empresa, se establecieron 36 variables estratégicas, identificando en la zona de enlace seis variables altamente influyentes y dependientes, correspondientes a: línea de productos, cobertura de distribución de mercado, rotación del inventario, logística de distribución, precios competitivos y administración de la fuerza de ventas.

El grado de convergencia y divergencia se identificó a través del método Mactor con la relación de 31 actores, destacando que la alianza más relevante es entre la Gerencia (GER) e Instituciones Financieras (INSF) y Jefe de Ventas (JEFV). Al considerar la correspondencia con los seis objetivos estratégicos y los involucrados, se plantea que tienen un compromiso alto con 30 posiciones valoradas a favor y 5 en contra para la gestión de una línea de harinas industriales con trigos importados, bajo la certificación World Basc Organization, ante el mismo objetivo la capacidad de los stakeholders es de 25 posiciones a favor y 5,8 en contra.

Con la utilización del sistema Smic Prob Expert se obtuvieron 64 escenarios posibles de ocurrencia, identificando dos escenarios importantes como es el tendencial (000000) que representa el 31,9\% equivalente al no cumplimiento de los retos estratégicos y la apuesta que cumple con todos los objetivos (111111), con un nivel de ocurrencia del 27,7 \% favoreciendo la gestión de una línea de harinas industriales con trigos importados, aumento de la cobertura de mercado, precios competitivos, adecuada administración a la fuerza de ventas, incremento de la rotación de mercado y planificación logística con la aplicación de un Sistema de Voice Picking.

\section{BIBLIOGRAFÍA}

Armijos, L., Galarza, S. y García, J. (2017). El pensamiento prospectivo y sus enfoques en las organizaciones latinoamericanas. Revista la Ciencia, 19 (3), 317-333. https://doi.org/10.24133/ ciencia.v19i3.538

Campos, W. y Salas, I. (2016). Variables estratégicas para el Quindío: un estudio de prospectiva territorial. Revista de Investigaciones de la Escuela de Administración y Mercadotecnia del Quindío EAM, $8(2), 1-21$.

Código Orgánico de la Producción, Comercio e Inversiones (2010). Registro Oficial Órgano del Gobierno del Ecuador. COPCI. https://bit.ly/2XFkHIb 
David, F. (2013). Conceptos de Administración estratégica (decimocuarta ed.). Pearson Educación.

Godet, M. y Durance, P. (2011). La prospectiva estratégica para las empresas y los territorios. Prospektiker. Instituto Nacional de Estadísticas y Censos (2010). Fascículo provincial Sto. Domingo. INEC. https:// bit.ly/2lWgr5G

Instituto Nacional de Estadísticas y Censos. (2012). Clasificación Nacional de Actividades Económicas. INEC. https://bit.ly/2YOjWF 1

Lezcano, E. (2010). Levaduras. Buenos Aires: Secretaría de Agricultura, Ganadería y Pesca. https://bit. ly/2S2fDb5

Mesa Editorial Merca2.0 (22 de 05 de 2015). Merca 2.0. Galería: 7 marcas que nos enseñan qué es la línea de productos. https://bit.ly/2XTYbqo

OIT, Organización Internacional del Trabajo (2016). Mejore su negocio. Comercialización. Ginebra: OIT. https://bit.ly/2Lusllw

Payehuanca, I. y Matos, A. (2011). La formación de la masa, la fermentación y los métodos de proceso en la elaboración del pan. I Congreso Nacional de Investigación (págs. 1- 8). Conacin. https://bit.ly/2XVMnUp

Porter, M. E. (2008). Las cinco fuerzas competitivas que le dan forma a la estrategia. Harvard Business Review.

Quiñonez, M. (2017). Procedimiento para incrementar la gestión competitiva de las pequeñas y medianas empresas comerciales ecuatorianas. Ciencias Administrativas, 3 (4), 364-383. https:// doi.org/10.23857/dom.cien.pocaip.2017.3.4.oct.364-383

Sampieri, R., Collado, C. y Baptista, P. (2010). Metodología de la Investigación (quinta ed.). Mc Graw Hill. 